\title{
HEURÍSTICAS Y SESGOS DE LOS ESTUDIANTES DE PRIMER CICLO DE UNIVERSIDAD EN LA RESOLUCIÓN DE PROBLEMAS DE PROBABILIDAD
}

\author{
GUISASOLA, JENARO ${ }^{1}$ y BARRAGUÉS, JOSÉ IGNACIO ${ }^{2}$ \\ ${ }^{1}$ Departamento de Física Aplicada I. Universidad del País Vasco \\ 2 Departamento de Matemática Aplicada. Universidad del País Vasco
}

\begin{abstract}
Resumen. Paralelamente al interés que recientemente está despertando la incorporación de la estadística y la probabilidad a los desarrollos curriculares, surge la evidencia de que existen obstáculos importantes para el aprendizaje significativo de los conceptos involucrados. Estas dificultades y concepciones alternativas están siendo investigadas sobre todo en alumnos de secundaria, pero no así en alumnos universitarios. El presente trabajo intenta, en primer lugar, detectar las concepciones de los estudiantes universitarios cuando tratan de explicar fenómenos aleatorios dentro de una teoría introductoria de la probabilidad. En segundo lugar, trata de agrupar estas concepciones en categorías explicativas que den una idea de la progresión del aprendizaje de los estudiantes.

Palabras clave. Azar y probabilidad, creencias previas, concepciones alternativas, aprendizaje significativo.
\end{abstract}

Summary. Along with the high interest created by the addition of Statistics and Probability into curricular projects arises the evidence of important obstacles in the meaningful learning of the different related concepts. These obstacles and the various alternative concepts are the subject for many studies, which mostly consider secondary students. Therefore, there is a lack of such research works for University undergraduate students. This work aims at finding what University undergraduate students understand by random phenomena, when the concept is part of an introductory probability theory. Secondly, it aims at grouping the students' alternative conceptions in self-explanatory categories, which hopefully will be of use for the understanding of the students' learning progress.

Keywords. Chance and probability, naive conceptions, misconceptions, significant learning.

\section{INTRODUCCIÓN}

Como indica Batanero (2000), sólo muy recientemente la probabilidad y la estadística han sido incorporadas al currículo de matemáticas de la enseñanza primaria y secundaria y de las diferentes especialidades universitarias en los países desarrollados. Las razones de este interés hacia la formación en probabilidad y estadística son de muy diversa naturaleza. De una parte, se considera que se trata de un área esencial en la formación de ciudadanos adultos capaces de orientarse en un entorno de fuertes interdependencias sociales, políticas y económicas, en las que se precisa interpretar gráficos de datos y en las que con frecuencia las decisiones se toman sobre la base de estudios estadísticos. Por otra parte, la probabilidad y la estadística contribuyen a aportar una imagen mucho más equilibrada de la ciencia, que tradicionalmente ha presentado ante el alumno un carácter marcadamente determinista, en la cual todo era explicable en términos de causas y efectos.

Razones como las apuntadas parecen ser impulsoras de la investigación en desarrollo curricular en el campo específico de la estadística y la probabilidad (Grupo Azarquiel, 1996; Díaz et al., 1996; Gómez y Perry, 1996; Ahlgren y Garfield, 1991). Sin embargo, también se ha señalado repetidamente que una de las mayores dificultades de los estudiantes en el aprendizaje de la teoría de la probabilidad y la estadística reside en el aprendizaje significativo de los conceptos (Borovenik et al., 1991; 
Batanero et al., 1997). Ello ha hecho que este aprendizaje sea una fuente constante de preocupación entre el profesorado y que el campo de los conceptos relativos al azar y la probabilidad se haya convertido en una de las áreas emergentes de investigación en la enseñanza de las matemáticas. En particular, las dificultades de aprendizaje de los conceptos de fenómeno aleatorio, frecuencia relativa, variabilidad de las pequeñas muestras, equiprobabilidad, independencia, etc., han sido investigadas detenidamente en el nivel de enseñanza secundaria (Batanero, 2000). Estos estudios evidencian la utilización de diversos mecanismos y estrategias por parte de las personas cuando deben emitir algún juicio en situaciones de incertidumbre provocada por el azar (Borovcnik et al., 1991; Scholz, 1991; Muñoz, 1998; Sáenz, 1998; Serrano et al., 1996, 1998). Así, se constatan las dificultades de los estudiantes para interpretar el valor de la probabilidad de un suceso (Saénz, 1998; Scholz, 1991; Serrano et al., 1996; Borovcnik et al., 1991; Borovnick y Peard, 1996) y a la hora de calcularla (Lecoutre, 1985, 1992; Lecoutre y Durand, 1988; Lecoutre y Cordier, 1990; Batanero et al., 1997; Konold, 1991).

Comprender conceptos como los de frecuencia relativa de un suceso, probabilidad de un suceso, relación entre ambos, equiprobabilidad, independencia, etc. es esencial si se quiere iniciar a los estudiantes en una visión matemática de los fenómenos de azar. En este sentido, entendemos por visión matemática aquélla que parte de la axiomática de Kolmogorov y emplea la terminología de la teoría de conjuntos para la definición de conceptos y la formulación de resultados. No obstante, los resultados del presente estudio se circunscriben a un marco teórico menos general, el frecuencial, en el que se establecen como prerrequisitos básicos, entre otros, saber reconocer la naturaleza aleatoria del fenómeno y justificar la adopción del modelo probabilístico frente a otro modelo, obtener el espacio muestral, definir formalmente el suceso, estudiar la independencia entre sucesos, calcular la probabilidad del suceso e interpretar frecuencialmente su valor. Debe aclararse que en este trabajo el concepto de probabilidad en sentido clásico (laplaciano) ha sido considerado como un caso particular del frecuencial (Gnedenko, 1996; Canavos, 1992). De este modo, la regla de Laplace puede aplicarse una vez establecida la hipótesis de equiprobabilidad de todos los sucesos elementales, admitiendo, por tanto, que cada uno de ellos aparece a largo plazo con una frecuencia relativa similar.

Todo lo anteriormente apuntado remarca la importancia de conocer con el mayor detalle bajo qué aspectos se presentan estas conceptualizaciones alternativas del azar y la probabilidad. Y es en este punto en el que creemos que esos mecanismos alternativos han sido escasamente investigados en estudiantes universitarios. En efecto, pocos estudios didácticos se han realizado sobre las ideas de los estudiantes respecto al modo de entender el azar y la probabilidad en primer ciclo de universidad. Es necesario investigar si también en este nivel educativo las creencias previas o adquiridas en la enseñanza por los estudiantes juegan un papel importante a la hora de abordar con éxito la enseñanza-aprendizaje de la proba- bilidad, tal como indica la investigación didáctica para la enseñanza secundaria (Díaz et al., 1996, Batanero, 2000; Kapadia, 1984).

En el siguiente apartado nos referiremos con mayor detalle al marco teórico en el que se circunscribe este trabajo-el paradigma de heurísticos y sesgos del razonamiento probabilístico, línea de investigación iniciada por los psicólogos Kahneman y Tversky-, que luego ha ampliado su campo de hipótesis y explicaciones. Nuestro objetivo ha sido intentar encontrar las explicaciones que los estudiantes universitarios proponen, tras haber recibido un curso probabilístico elemental, en situaciones relacionadas con los conceptos mencionados.

\section{MARCO TEÓRICO}

La investigación sobre las intuiciones de los individuos acerca del azar y la probabilidad cuenta con una larga tradición en psicología y en didáctica de la probabilidad (Borovcnik y Bentz, 1991; Scholz, 1991). Desde Piaget, en los años cincuenta, los psicólogos vienen describiendo diversos tipos de pensamiento probabilístico y diseñando teorías con las que estudiar su desarrollo en el curso de maduración del individuo. El interés que estas investigaciones poseen para la enseñanza de la probabilidad viene siendo remarcado insistentemente en la bibliografía (Kapadia, 1984).

Existe evidencia del uso de diversos mecanismos y estrategias no probabilísticas por parte de las personas cuando deben emitir algún juicio acerca de situaciones no deterministas (Muñoz, 1998; Sáenz, 1998; Serrano et al., 1996, 1998). Estas estrategias o heurísticos se emplearían para reducir la incertidumbre que produce la propia limitación a la hora de enfrentarse a la complejidad de los estímulos ambientales.

Estas estrategias son adquiridas a través de las experiencias de la vida cotidiana, tienen un valor práctico, es decir, son mecanismos de enjuiciamiento o decisión, y son persistentes y sistemáticas. Forman, por tanto, un cuerpo de conocimientos, una representación mental consistente (Pozo, 1999). El problema es que pueden impedir o sesgar la aplicación de los conceptos matemáticos acerca del azar.

Afortunadamente las investigaciones recientes prueban que los juicios probabilísticos que los individuos realizan están dirigidos por el uso sistemático de unos pocos patrones de inferencia (Kahneman et al., 1982). Seguidamente revisaremos algunos de los patrones que, según la bibliografía citada, más frecuentemente aparecen en los estudiantes (Scholz, 1991; Muñoz, 1998; Sáenz, 1998; Serrano et al., 1996, 1998).

\section{Heurística de accesibilidad}

Consiste en estimar la probabilidad de un suceso según la facilidad con que se recuerdan ejemplos en los que 
dicho suceso ocurrió o por la facilidad con que pueden generarse ejemplos en los que tal suceso ocurre (Sáenz, 1998; Scholz, 1991; Hirsch y O'Donnell, 2001). Paulos (1995) señala que la literatura psicológica contiene una gran cantidad de trabajos sobre el error de la accesibilidad o disponibilidad y que se trata de un fenómeno ampliamente difundido en los medios de información. Descrito en primera instancia por los psicólogos Tversky y Kahneman, consiste, como ya se ha indicado, en la fuerte disposición a «emitir juicios o valoraciones a la luz de lo primero que pasa por la cabeza o que está disponible en la cabeza».

\section{Heurística de representatividad}

Bajo esta concepción, se estima la probabilidad de un suceso de acuerdo con lo representativo de cierta población que parece ser dicho suceso (Sáenz, 1998; Serrano et al., 1998). Kahneman y Tversky (1972) exploraron lo que ellos llamaron «heurísticas de la representatividad», esto es, la asignación de probabilidades altas a aquellos sucesos que parecen ser prototípicos de una población y bajas probabilidades a los que parecen no serlo. En palabras de Kahneman y Tversky, «una persona que sigue este patrón evalúa la probabilidad de un suceso o de una muestra según el grado en que cuenta con propiedades similares a las de la población de donde proviene, y refleja las características del proceso mediante el cual ha sido generado».

Existen varias concepciones erróneas que surgen comúnmente de la heurística de la representatividad. Dos de ellas son la insensibilidad al tamaño de la muestra y diversas ideas erróneas acerca de las secuencias aleatorias (Serrano et al., 1998).

\section{Insensibilidad al tamaño de la muestra}

Conduce a pensar que cualquier muestra debe reproducir todas las características de la población, aunque se trate de una muestra de pequeño tamaño. En palabras de Serrano y otros (1998), parece creerse una «ley de los pequeños números», según la cual las pequeñas muestras serían representativas de todas las características de la población de la cual provienen y, en consecuencia, no se toma en consideración la importancia del número de ensayos en las estimaciones frecuenciales de probabilidad.

\section{Concepciones erróneas sobre las secuencias aleatorias}

Existe la creencia de que las secuencias de resultados que aparecen relativamente ordenadas o simétricas no pueden considerase aleatorias «por no tener aspecto aleatorio» (Stewart, 1998). Estas ideas conducen, por ejemplo, a que se considere poco probable un número de la lotería como 12345.

\section{El sesgo de equiprobabilidad}

Se refiere a la creencia de los sujetos en la equiprobabilidad de todos los sucesos asociados a cualquier experimento aleatorio, incluso en aquéllos en que no es aplica- ble el principio de la indiferencia. Lecoutre (1985, 1992), Lecoutre y Durand (1988) y Lecoutre y Cordier (1990), estudiando el problema, preguntaron a los sujetos qué es más probable al lanzar dos dados, «obtener un cinco y un seis» o bien «obtener dos seises». A pesar de variar el contexto de la pregunta, el formato, la edad y la formación de los sujetos, existe una coincidencia que muestra la gran estabilidad en la creencia de que ambos sucesos son equiprobables.

\section{El sesgo determinista}

Un razonamiento causal es aquél que asocia causas y efectos a los fenómenos que acontecen. Una concepción causal espontánea responde a una determinada visión del mundo, a un modo de ver y explicar cómo se desarrollan los acontecimientos, visión que está presente en la manera habitual con que las personas se expresan y en los mecanismos de acción que describen (Solsona et al., 2000). Las dependencias físicas cuentan con referente cotidiano en la experiencia humana y son también ingredientes del aprendizaje social («si haces esto, entonces conseguirás aquello»). Se dispone de todo un cuerpo de causas que dan lugar a una consecuencia bien definida. Borovcnik y Bentz (1991) y Borovcnik y Peard (1996) señalan que los tipos de razonamiento lógico, causal y probabístico están jerárquicamente ordenados. Si se encuentra una razón lógica, entonces es aplicada de inmediato, incluso aunque fuera posible usar también cierto argumento causal que haya sido empleado anteriormente. El argumento probabilístico cuenta con el menor nivel de aceptación.

\section{Enfoque del resultado aislado}

En lugar de explorar cómo los sujetos realizan los juicios de probabilidad, diversos autores se han interesado por el modo en que se interpretan las preguntas sobre probabilidad o sobre el significado que se atribuye al valor de la probabilidad (Konold, 1991). La conclusión ha sido que en ocasiones se interpreta una pregunta sobre probabilidad de forma no probabilística. En concreto, la probabilidad de un suceso puede interpretarse como la formulación de una predicción acerca de si tal suceso ocurrirá o no en el siguiente experimento. Las personas que presentan este enfoque evalúan las probabilidades comparándolas con los valores $0 \%, 50 \%$ y $100 \%$. Si una probabilidad se acerca a los extremos $0 \%$ o $100 \%$, el suceso se considera imposible o seguro, respectivamente. Sólo si se acerca al $50 \%$ se considerará verdaderamente aleatorio, tendiendo a buscar explicaciones causales en lugar de aleatorias a la ocurrencia de resultados inesperados y a la variabilidad de los fenómenos aleatorios. Las investigaciones sugieren que los alumnos que muestran la concepción de la probabilidad como pronóstico consideran que cada una de las repeticiones de un experimento aleatorio no tiene por qué guardar relación con las anteriores o posteriores; en consecuencia, podrían tener dificultad para comprender un enfoque frecuencial de la probabilidad en la enseñanza (Serrano et al., 1998).

A partir de un análisis de los contenidos y las habilidades cognitivas que son necesarios para comprender de forma 
significativa la teoría de la probabilidad a nivel introductorio así como de la revisión de la bibliografía citada, parece plausible plantear como hipótesis que, tras un curso universitario elemental sobre teoría de la probabilidad, en la mayoría de los estudiantes se detectarán los sesgos estadísticos descritos, que constituyen ideas alternativas a las formales acerca del azar y del modo de estimar la probabilidad y de interpretar su significado. Contrastar esta hipótesis ha sido el primer objetivo de este trabajo. El segundo objetivo consiste en analizar el modo en que el contenido de la tarea propuesta influye en la estrategia de solución adoptada por el alumno (Borovcnik y Bentz, 1991).

\section{DISEÑO EXPERIMENTAL}

Para contestar a los interrogantes planteados se ha ideado un diseño múltiple y convergente que incluye métodos cualitativos (entrevistas) y cuantitativos (encuestas). En el diseño se ha incluido un cuestionario con 12 preguntas de tipo abierto con énfasis en las explicaciones y entrevistas estructuradas a una muestra de estudiantes. En las entrevistas se trata de observar si se contrastan de forma cualitativa y convergente los análisis de resultados obtenidos en los cuestionarios.

El cuestionario se pasó a un total de 110 estudiantes, distribuidos en tres grupos de $2^{\circ}$ curso de Ingeniería Técnica Industrial que fueron elegidos de forma aleatoria. En los currículos de estas titulaciones se incluye, en este curso, una asignatura cuatrimestral dedicada a la estadística y al cálculo de probabilidad elementales y, en primer curso, sendas asignaturas dedicadas al cálculo infinitesimal y al álgebra lineal. Todos los grupos habían recibido enseñanza sobre el tema de probabilidad en sus respectivos cursos impartida por profesores competentes y con experiencia.

El cuestionario fue pasado por el mismo investigador a todos los grupos cuando los estudiantes habían terminado el estudio del tema. Los estudiantes respondieron al cuestionario bajo condiciones de examen (sin comunicarse con otros estudiantes) durante una clase de 55 minutos. Como el cuestionario resultaba excesivamente largo para una sesión, se dividió en dos cuidando el equilibrio en la dificultad, y se repartió aleatoriamente. Hemos de indicar que, de forma general, los estudiantes no mostraron problemas para entender los enunciados de las cuestiones.

\section{Instrumentos de evaluación}

En un trabajo previo (Guisasola y Barragués, 1999) se analizaron las explicaciones que daban los estudiantes de 1r. curso de Ingeniería Técnica Industrial sobre fenómenos de aleatorios estudiados en un tema de introducción a la teoría de la probabilidad. Allí se constató en una muestra pequeña (30 alumnos) que las interpretaciones de los estudiantes sobre estos fenómenos presentaban una baja coherencia global, pero mayoritariamente se expresaban ideas que se encontraban fuera del marco teórico formal de la probabilidad, tendiendo a razonar en términos causales y recurriendo a su experiencia personal para estimar la probabilidad de un suceso, todo ello en consonancia con lo que recoge la bibliografía especializada.

Después de una revisión de los primeros resultados obtenidos en el estudio limitado que hemos mencionado, en el trabajo que aquí presentamos hemos realizado una investigación más sistemática orientada hacia estudiantes de $2^{\circ}$ curso de Ingeniería Técnica Industrial, que es el curso en el que se imparte un cuatrimestre específico de Probabilidad y Estadística, de acuerdo con el plan de estudios.

En el diseño para este trabajo, el formato de cuestionario de preguntas ha sido elegido como el más apropiado para indagar en una muestra de 110 estudiantes. Para aumentar la efectividad del cuestionario hemos utilizado preguntas cualitativas y de formato abierto. Los objetivos de los 12 ítems del cuestionario abarcan contenidos conceptuales relacionados con las características de los fenómenos aleatorios y la estimación de la probabilidad (ítems 1 a 8) y con la interpretación frecuencial de probabilidad (ítems 9 a 12).

La indagación sobre cada uno de los aspectos ha sido realizada diseñando más de un ítem, ya que hemos tenido en cuenta que los estudiantes pueden responder de forma diferente según el tipo de tarea propuesto. Esta diversidad de ítems para analizar el mismo objetivo de aprendizaje nos ha permitido contrastar el mismo problema en diferentes situaciones, con lo cual se obtiene un análisis más coherente de las características del conocimiento de los estudiantes.

Los contenidos y objetivos de las cuestiones han sido validados por investigadores expertos en el campo de la probabilidad y por profesores con experiencia en la investigación didáctica.

Para analizar las respuestas del cuestionario se ha tenido en cuenta la revisión exhaustiva de las preguntas realizada por un miembro del equipo de investigación de la que se derivó un conjunto de categorías para cada cuestión. Las respuestas de los estudiantes eran clasificadas de acuerdo con las categorías definidas. Una vez que las categorías fueron discutidas con otros dos componentes del equipo de investigación se realizó una sesión de entrenamiento en la que se examinó el $10 \%$ de la muestra. A continuación los miembros del equipo de investigación procedimos a analizar independientemente todos los cuestionarios. El nivel de acuerdo entre los correctores a la hora de clasificar las respuestas fue muy alto en todas las cuestiones. En los casos de desacuerdo, la categorización definitiva se realizó mediante discusión y consenso entre los tres correctores. Una vez realizado el análisis se encontró que las frecuencias de las categorías de respuesta se mantenían para aquellas cuestiones con el mismo objetivo y similar dificultad. 
La segunda prueba ha consistido en entrevistar ante varios fenómenos aleatorios a un total de 15 estudiantes de $2^{\circ}$ curso de Ingeniería Técnica Industrial. Las entrevistas tienen como objetivo ver cómo explican los estudiantes cuatro situaciones problemáticas sobre fenómenos aleatorios y contrastar si estas explicaciones se aproximan o no a la teoría de probabilidad estudiada en clase. Las entrevistas han tenido una duración media de 30 minutos y han sido grabadas en cinta magnetofónica para su posterior transcripción y análisis. La estructura de la entrevista consiste en que el estudiante se plantee el fenómeno y piense sobre lo que sucede, estimulándole a que emita conjeturas lo más fundamentadas posible (White y Gunstone, 1992).

\section{RESULTADOS Y DISCUSIÓN}

Para facilitar la presentación y la discusión de los resultados obtenidos, los indicaremos en diferentes secciones y los ilustraremos con resultados de los cuestionarios así como con resultados cualitativos de las entrevistas.

\section{¿Qué entienden los estudiantes por fenómeno aleato- rio?}

En el ítem 1 se definen los sucesos $S_{i}$ tales que $p\left(S_{i}\right)$ es desconocida. Se trata de calcular la probabilidad de cierto suceso, marcando una de las respuestas y explicando cuál ha sido su razonamiento. Son varias las respuestas que pueden darse dentro del marco teórico. Es posible formular de forma explícita alguna hipótesis sobre la probabilidad de que suban las acciones de cada una de las tres empresas y, seguidamente, calcular la probabilidad. También puede no formularse hipótesis alguna y operar con cierto número de parámetros, ofreciendo una solución no numérica. Finalmente, una respuesta de menor calidad de razonamiento sería aquélla que explica que las probabilidades de que suban las acciones de cada empresa son desconocidas y, en consecuencia, no es posible calcular la probabilidad pedida.

Con este ítem tratamos de detectar los sesgos de equiprobabilidad y determinista. Respecto al primero, debemos precisar que en las fases previas de la investigación ensayamos con enunciados de tipo similar al ejemplo que hemos citado en la revisión teórica. Sin embargo, cuando nuestros alumnos comprendían el enunciado en los términos que deseábamos (esto es, el suceso «obtener un 5 y un 6» quiere decir «obtener cualquiera de los resultados 5-6 o 6-5»), no observamos en ellos el sesgo de equiprobabilidad (esto es, los alumnos respondían correctamente que $\mathrm{p}(66)<\mathrm{p}(5-6$ o 5-6)). En consecuencia, debimos construir nuestro ítem 1 con el que no tratamos de observar en los alumnos la creencia en la equiprobabilidad de todos los sucesos (subconjuntos del espacio muestral) sino en la de todos los resultados del experimento (elementos del espacio muestral). Esta creencia conduce en la situación planteada a asumir de forma implícita la equiprobabilidad de la subida y bajada del valor de las acciones de cada empresa y, en consecuen- cia, la equiprobabilidad de los ocho elementos del espacio muestral.

Respecto al sesgo determinista, buscamos respuestas del tipo «el fenómeno es demasiado complejo para ser estudiado», que, como indican Serrano y otros (1996) y Borovcnik y Bentz (1991), parecen revelar una interpretación causal de los fenómenos aleatorios. Nosotros también creemos que es plausible atribuir al sesgo determinista este tipo de respuesta, ya que el sujeto que trata de resolver los problemas en términos de causas y efectos puede apreciar la imposibilidad de aplicar este tipo análisis en fenómenos complejos. El problema es que este mismo sujeto no estaría comprendiendo que la solución probabilística busca un tipo de regularidad cualitativamente diferente a la determinista, independientemente de que ésta pueda o no obtenerse y sea o no apropiada.

He aquí algunos ejemplos de este tipo de razonamiento:

«Teniendo en cuenta cómo es la bolsa, es muy difícil de calcular la probabilidad de que suban las acciones.»

«Este tipo de probabilidad no se puede calcular con certeza. Si no, nadie perdería dinero.»

«No se puede calcular porque la subida o la bajada de acciones no dependen de sí mismas sino de otras muchas cosas.»

Los resultados obtenidos para el ítem 1 se recogen en la tabla I.

Tabla I

Porcentajes de respuesta en el ítem 1.

\begin{tabular}{|l|c|}
\hline Categoría de respuesta & \% de respuestas $(\mathbf{N}=\mathbf{7 5})$ \\
\hline Respuesta correcta & 16 \\
\hline Sesgo de equiprobabilidad & 52 \\
\hline Sesgo determinista & 20 \\
\hline Cálculo erróneo de la probabilidad & 2,7 \\
\hline Respuesta incodificable & 1,3 \\
\hline En blanco & 8 \\
\hline
\end{tabular}

Las explicaciones de los estudiantes de la categoría «Respuesta correcta» consistieron en analizar el problema desde el marco teórico de la probabilidad explicado en clase y concluir que, al no conocer las probabilidades de los sucesos elementales, no pueden calcular la del suceso pedido. Ningún estudiante propuso una respuesta de mayor poder explicativo como las apuntadas al principio de la discusión. En la categoría «Cálculo erróneo de la probabilidad» se agrupan aquellas respuestas que enfocan correctamente el problema al formular explícitamente la hipótesis de equiprobabilidad de los sucesos «subir»y «bajar» para cada empresa pero luego cometen algún error en el cálculo de la probabilidad. 
Si el ítem 1 nos ha servido para detectar un primer indicio de la existencia del sesgo determinista, que como se ha visto consiste en la renuncia a cualquier tipo de estudio en aquellos fenómenos complejos en los que se perciba el azar, la primera situación problemática para una entrevista puede servir para dejar al descubierto un segundo indicio de este mismo sesgo: la confusión entre independencia causal e independencia estocástica (Falk, 1983). Obsérvese el siguiente fragmento de la entrevista a Fernando.

\section{- Fragmento de entrevista correspondiente a la situa- ción problemática 1}

Entrevistador: «La probabilidad de la primera carta, ¿ se modifica si tenemos el dato de lo que ha salido en la segunda extracción?»

Fernando: «Yo creo que no.»

$E$ : «¿Por qué?»

$F .:$ «Al tenerla apartada, que luego saques un as, esa carta no entra dentro ya del conjunto, desde el momento en que la hemos separado...»

$E .:$ «Entonces, das por buena esa primera interpretación que aparece propuesta.»

$F .: \ll$ Sí.»

$E$ : «La segunda interpretación es que ahora la probabilidad de que la primera carta haya sido un as es mayor que $1 / 2$.»

$F .:$ «Eso no.»

$E .:$ «Y la tercera interpretación, que la probabilidad es ahora menor.»

$F .:$ No.»

$E .:$ «Estonces, tú tienes una nueva información [la segunda carta es un as], pero dices que eso no altera la probabilidad del primer suceso [la primera carta es un as].»

$F .:$ «Claro, porque ocurrió antes, y ya está fuera de lo que estamos jugando ahora.»

$\mathrm{Si}$, como el alumno, se interpreta el enunciado en términos causales, resulta evidente que el resultado de la segunda extracción no afecta al resultado de la primera extracción. Así pues, la nueva información no afecta a la probabilidad de la primera extracción. Pero el conflicto surgirá poco después en la entrevista, cuando el profesor hace observar que, si se van descubriendo nuevas cartas, lo que primeramente era incertidumbre (el resultado de la primera extracción) pasa a ser certeza.

- Fragmento de entrevista correspondiente a la situación problemática 1 (continuación)

$E .:$ «Fíjate en que lo que antes era incertidumbre se convierte ahora en certeza. Entonces, este cambio, ¿te parece que es directo de probabilidad $1 / 2$ a certeza o se va modificando a medida que yo voy sabiendo más cosas?»

$F .:$ «Claro, porque no ha sido lo mismo que me sacases primero un as y luego el otro as, que primero me sacases un as y luego un rey. Entonces sí que se modifica...»

$E$ : «Dices que hay una modificación de probabilidad. Pero todavía tenemos el problema de antes. La segunda carta no afecta a la primera. ¿Cómo podría modificar su probabilidad?»

$F .:$ [Se muestra desconcertado y no acierta a dar explicación alguna.]
Así pues, la probabilidad de la primera carta sí resulta de alguna forma alterada si se dispone de nuevos datos. ¿Cuál es la explicación? En todas las entrevistas realizadas, los alumnos perciben el conflicto pero no encuentran explicación ni relación con la probabilidad condicionada, que sería el análisis correcto desde un marco teórico formal de la probabilidad.

Como se ha visto, el sesgo determinista en los alumnos ha sido investigado de un modo indirecto, ya sea buscando respuestas del tipo «se trata de azar, no puede estudiarse el fenómeno» (ítem 1) o bien detectando confusión entre condicionamiento y causación (primera situación problemática para la entrevista). El enfoque del ítem 2 es diferente: se trata de detectar el sesgo de un modo directo. Más concretamente, el objetivo es detectar la creencia de que cualquier fenómeno queda unívocamente determinado por cierto conjunto de condiciones iniciales establecidas y que es posible predecir el estado final exacto sin más que aplicar las leyes físicas adecuadas (Borovcnik y Bentz, 1991). En el enunciado se describe el aparato de Galton, similar al que apareció en Natural Inheritance en 1869 (Díaz et al., 1996). El estudiante debe dar su opinión acerca del enfoque propuesto y explicar, en su caso, algún enfoque alternativo. Es de señalar que, para construir el enunciado del ítem 2 , hemos aprovechado que nuestros alumnos poseen un bagaje suficiente en materias técnicas, algo que no ocurre en secundaria. Los resultados se han agrupado en categorías de acuerdo con la tabla II.

En la categoría «Respuesta correcta» se han agrupado aquellas respuestas que desaconsejan razonadamente la solución propuesta, el tratar de predecir de forma unívoca en qué orificio entrará la bola, dada la gran cantidad de factores de naturaleza aleatoria que influirán en su posición final. De acuerdo con el marco teórico, en lugar del enfoque propuesto debe optarse por uno estocástico, esto es, se debe proponer una solución probabilística donde se estime o se calcule $\mathrm{p}(\mathrm{i}), \mathrm{i}=1 \ldots, 7$, siendo $p(i)$ la probabilidad de que la bola entre en el orificio $i$-ésimo.

Es necesario resaltar que todas las respuestas contenidas en esta categoría de «Respuesta correcta» desaconsejan claramente la solución propuesta, pero no entran en detalles ni cálculos formales. Este tipo de razonamiento se ilustra a continuación con un ejemplo:

Tabla II

Porcentajes de respuesta en el ítem 2.

\begin{tabular}{|l|c|}
\hline Categoría de respuesta & \% de respuestas (N = 67) \\
\hline Respuesta correcta & 16,4 \\
\hline Sesgo determinista & 38,8 \\
\hline Errores procedimentales & 29,9 \\
\hline Respuesta incodificable & 1,5 \\
\hline En blanco & 13,4 \\
\hline
\end{tabular}


«Teóricamente, utilizando únicamente las leyes físicas nos debería salir el lugar donde la bola caería, pero eso no es así, ya que, en la caída de ésta, intervienen factores externos no medibles a priori que condicionan la caída. Por ello lo mejor sería por cálculo de probabilidades: hacer una media ponderada entre el cálculo de probabilidades y de las condiciones físicas.»

Nótese que el alumno es capaz de hacer referencia a los principios físicos que, en una solución idealizada, podrían dar lugar a una respuesta exacta. Sin embargo, es también consciente de la dificultad de puesta en práctica de este modelo en la situación física real y, en consecuencia, cree que debería optarse por una solución probabilística.

Casi un $40 \%$ de las respuestas se sitúa en la categoría «Sesgo determinista», en las que el alumno acepta explícitamente la solución propuesta, sin tomar en consideración la naturaleza del problema. Los siguientes son ejemplos de este tipo de respuesta:

«Controlando todas las características físicas de todos los objetos y sabiendo qué dirección, aceleración, etc. va a llevar la bola en el punto de partida, creo que se podría saber dónde acabará la bola. Todos los detalles son de importancia, pues requieren gran exactitud: clavos (tamaño, orientación en que han sido clavados, profundidad...), bola, fuerza y orientación del lanzamiento de la bola, y otras muchas características, como flexibilidad de los clavos y de la bola para saber posibles respuestas ante choques, etc.»

«En primer lugar es muy importante la precisión en el experimento, por lo que las medidas, etc. deben ser muy correctas. Siendo la posición de los clavos exacta, se podrán aplicar las leyes físicas. Emplear material preciso, que la bola sea homogénea, etc.; precisión en la colocación de los clavos...Si todo está calculado y situado de forma precisa, la bola siempre caerá hacia un lado, debido a la rotación de la Tierra (lo mismo que ocurre con el agua que cae de los grifos).»

Alrededor de un tercio de los estudiantes (categoría «Errores procedimentales») elige un enfoque probabilístico como posible solución, pero no explica las razones de esta elección, es decir, no analiza cuáles serían las dificultades del enfoque determinista que se propone en el enunciado. Estas respuestas no contemplan uno de los principales aspectos procedimentales a la hora de analizar un fenómeno como es la valoración de la naturaleza aleatoria del mismo. Ejemplos de este tipo de respuesta se indican a continuación:

«No hay mucha física para aplicar. Anotar cuidadosamente los resultados obtenidos y en función de éstos elaborar una tabla de probabilidades.»

«Cuando la bola se deja caer y rebota en un clavo sólo tiene dos opciones, derecha e izquierda, con la misma probabilidad. Habrá que mirar por cuántos caminos llega la bola a cada agujero. Sumamos todos los caminos posibles y se obtienen que para el núm. 4 hay 20 , para los núm. 3 y 5 hay 15 , para los núm. 2 y 6 hay 6 y para los núm. 1 y 7 hay $1 . »$

Como indican Serrano y otros (2001), la percepción subjetiva de la aleatoriedad por parte de sujetos adultos ha sido investigada a través de dos tipos principales de tareas. El primer tipo consiste en pedir a los sujetos que escriban una secuencia de resultados que esperarían encontrar al repetir cierto número de veces un experimento aleatorio. El segundo tipo consiste en ofrecer a los sujetos una serie de secuencias de resultados y pedir su opinión acerca de su posible aleatoriedad. Las tareas de los ítems 3 y 4 corresponden a este segundo tipo y tienen como objetivo principal detectar en los estudiantes el uso del criterio de presencia o ausencia de patrones o simetrías fácilmente reconocibles para tomar una decisión acerca de la aleatoriedad de la secuencia.

Es de señalar que los alumnos en ambos ítems deben emitir un juicio de confianza acerca de la equiprobabilidad de los sucesos elementales, de modo que parecen referirse al marco teórico de la probabilidad subjetiva y no al frecuencial. Ambos ítems (y todos los demás) pueden tener, en efecto, una respuesta dentro de un marco teórico que contemple la probabilidad subjetiva, pero también la tienen en el marco frecuencial, que es con el que contaban nuestros alumnos. Así, cuando deben emitir un grado de confianza, no se trata de que evalúen una probabilidad, sino de que analicen a la luz del marco frecuencial si es o no posible emitir un juicio acerca de la equiprobabilidad de los sucesos elementales.

Los criterios de empleados para valorar las respuestas son, en efecto, frecuenciales (ver discusión de resultados más abajo). Precisamente las secuencias propuestas son muy cortas a fin de detectar otra de las concepciones erróneas que surgen de la heurística de la representatividad: insensibilidad al tamaño de la muestra. Borovenik y Bentz (1991), Hirsch y O'Donnell (2001) y otros autores han empleado también secuencias muy cortas como las de nuestros ítems, mientras que Serrano y otros (2001) han empleado secuencias más largas y han identificado otras categorías sobre la percepción de la aleatoriedad, como es la alternancia. La escala para los valores de confianza (de 0 a 4 ) que aparece en los ítems 3 y 4 no tiene otro objetivo que definir con precisión qué significado debe atribuir el alumno a cada uno de los valores de confianza propuestos.

Para el ítem 3 las respuestas de los estudiantes se han agrupado de acuerdo con las categorías que aparecen en la tabla III.

Según el marco teórico, una respuesta se considera correcta si señala confianza (2) en cada una de las secuencias, explicando que la muestra de ocho datos no es representativa para tomar una decisión probabilísticamente razonada. Ningún alumno ofreció una respuesta correcta.

Más del 80\% de las respuestas se encuentra en la categoría «Ideas incorrectas acerca de las secuencias aleatorias». Estas respuestas explican que los fragmentos de una muestra en los que se reconoce alguna clase de orden (secuencias 1, 3 y 5) se suponen menos frecuentes que aquellos otros de igual tamaño en los que no se aprecia orden alguno (secuencias 2 y 4). Ejemplos de esta categoría de respuesta son los siguientes: 
Tabla III

Porcentajes de respuesta en el ítem 3.

\begin{tabular}{|l|c|}
\hline Categoría de respuesta & \% de respuestas $(\mathbf{N}=\mathbf{3 2})$ \\
\hline $\begin{array}{l}\text { Ideas incorrectas acerca } \\
\text { de las secuencias aleatorias }\end{array}$ & 81,3 \\
\hline Respuesta incodificable & 6,3 \\
\hline En blanco & 12,4 \\
\hline
\end{tabular}

Óscar, marca confianza 1 en la ruleta 3: «Parece mucha coincidencia el que vayan alternando $\mathrm{R}$ y $\mathrm{N}$.»

Jesús Maria, marca confianza 1 en la ruleta 1 : «Porque es muy difícil obtener las cuatro primeras $\mathrm{R}$ y después N.»

Mikel, marca confianza 4 en la ruleta 4: «Es un resultado muy aleatorio $\mathrm{y}$, por tanto, probable.»

Shaughnessy (1983), Green (1982), Borovcnik y Bentz (1991) y Muñoz (1998) se refieren a la dificultad de muchos individuos para diferenciar entre un resultado específico y todo un conjunto de resultados agrupados bajo cierta característica. Por ejemplo, para distinguir entre el resultado «12472» y «cualquier resultado obtenido con los dígitos $1,2,2,4,7$ ». Para investigar esta dificultad y mostrar cómo puede ser la base de un argumento empleado por los alumnos para sostener que un resultado «simétrico» como el 12345 es menos probable que otro «desordenado» como el 83056 , se ha diseñado la situación problemática 2 para una entrevista personal. He aquí dos fragmentos de sendas entrevistas realizadas a Itziar y a Fernando.

\section{- Fragmento de entrevista correspondiente a la situa- ción problemática 2}

Itziar: «Me fío más del aspecto.»

Entrevistador: «¿Por qué eliges el 83056?»

I.: «Hay muchos más números que no van seguidos, que los números que van seguidos. O sea, tengo 12345,23456 , así $x$ números. Pero de los que no van seguidos hay muchísimos más.»

- Fragmento de entrevista correspondiente a la situación problemática 2

\section{Ricardo: «Elegiría el último.»}

Entrevistador: «Vamos a hablar de tus razones. ¿Por qué ese número?»

$R .:$ «El primero lo descarto porque, si la primera bola es un 2, para que luego te vuelva a salir el mismo número... En el 83056 no se repite ninguno.»

E.: «Pero devuelves la bola. Posible sí es el 22211.»

$R .:$ «Sí, pero la probabilidad de volver a repetir el mismo número es mínima.»

E.: «¿Y el número 12345?»

$R$ : «Lo mismo, de que salgan ordenados. La probabilidad de que te salgan los números continuados es bastante mínima. Igual que 54321, que salgan los cinco números ordenados sería bastante difícil.»
Ningún alumno entrevistado recurrió a un análisis formal, sino que directamente se considera poco probable todo número que muestre alguna pauta, lo cual es convergente con los resultados del ítem 3 . Se detecta también la dificultad apuntada para distinguir entre un resultado específico y todo un conjunto de sucesos (Itziar, por ejemplo, argumenta con el suceso «dígitos no seguidos»).

Esta forma de razonar basada en ideas erróneas acerca de las secuencias aleatorias, que afecta a una gran mayoría de respuestas del ítem 3 (81\%), se encuentra alejada del marco teórico de la probabilidad explicado en clase. En todas estas explicaciones se manifiesta una «insensibilidad al tamaño de la muestra», no se toma en consideración que, en términos de teoría de la probabilidad, no se puede emitir un juicio acerca de la equiprobabilidad de un suceso sobre la base de la muestra de ocho datos.

Es de señalar que los alumnos pueden aceptar que es posible que cada una de las secuencias mostradas hayan sido obtenidas de forma aleatoria. Pueden encontrar evidente que, por ejemplo, es posible jugar ocho veces con una ruleta legal y obtener ocho negros. Pero las ideas erróneas acerca de cuál debe ser el aspecto de una secuencia aleatoria o acerca del número esperado de ocurrencias de un suceso conducen a considerar que una secuencia como NNNNNNNN es «posible, pero improbable»y, en algunos casos, los alumnos juzgan a esa secuencia como «muy difícil, casi imposible». En ningún momento toman en consideración que, bajo equiprobabilidad, la probabilidad de toda secuencia específica es la misma y que, si se trata de contrastar precisamente la hipótesis de equiprobabilidad (como es el caso de este ítem), debe contarse con una muestra lo suficientemente amplia.

En el ítem 4 se presenta una situación similar a la del ítem 3 , pero ahora siguiendo un esquema bidimensional parecido al empleado por Serrano y otros (1999). También en este caso hemos propuesto secuencias muy cortas a fin de detectar una insensibilidad al tamaño de la muestra. Los resultados son similares a los del ítem anterior, el $88,6 \%$ de las respuestas se encuentra en la categoría «Ideas incorrectas acerca de las secuencias aleatorias». Estas respuestas consideran que sí es posible tomar una decisión acerca de la probabilidad de cada casilla con los datos disponibles y se emplean las simetrías visibles en los casilleros como base para asignar el grado de confianza. Nótese que este criterio de decisión se emplea a pesar de lo irrelevante del hecho de que la secuencia elegida por la máquina presente o no una estructura fácilmente reconocible, ya que el jugador apuesta por una sola casilla.

\section{¿Cómo estiman los estudiantes la probabilidad de un suceso?}

En el ítem 5 se describen dos poblaciones $P$ (trabajadores de banca) y $Q$ (miembros de alguna ONG), presumiblemente no disjuntas. El estudiante debe razonar qué es más probable que el elemento sea sólo un trabajador de 
la banca (pertenezca a $P$ ) o que, además de trabajar en la

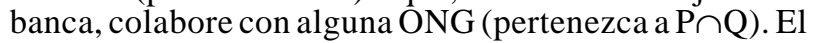
objetivo del ítem es detectar una concepción de la probabilidad según la cual la probabilidad de que cierto elemento pertenezca a cierta población se estima atendiendo solamente a la experiencia personal acerca de ambos, esto es, considerando sólo el grado en que el elemento se considere típico de la población, sin ningún intento de análisis formal (heurística de la representatividad). Los resultados obtenidos se muestran en la tabla IV.

Tabla IV

Porcentajes de respuesta en el ítem 5.

\begin{tabular}{|l|c|}
\hline Categoría de respuesta & \% de respuestas $(\mathbf{N}=\mathbf{4 8})$ \\
\hline Respuesta correcta & 16,7 \\
\hline Heurística de la representatividad & 77,1 \\
\hline Respuesta incodificable & 4,2 \\
\hline En blanco & 2,1 \\
\hline
\end{tabular}

En la categoría «Respuesta correcta» se han agrupado aquellas respuestas que razonan de acuerdo con el marco teórico. Esto implica un análisis del modo en que se han construido los sucesos 1 y 2 . Así pues, se debe observar que 2 es más restrictivo que 1 , es decir, $(2) \subset(1)$, con lo cual $p(2) \leq p(1)$. Sin embargo, el número de respuestas $(16,7 \%)$ se puede considerar muy bajo.

Más de las tres cuartas partes del total de respuestas se encuentran en la categoría «Heurística de la representatividad». En esta categoría, las explicaciones estiman la mayor o menor probabilidad de que cierto elemento $C$ pertenezca a cierta población $S$, atendiendo solamente a la experiencia personal acerca de las características de $C$ y de $S$ sin ningún intento de análisis formal y al margen del marco teórico explicado en clase. Así, se razona que es más probable el suceso 2 porque el perfil de «persona joven, soltera, abierta, brillante, universitaria y muy interesada en las cuestiones sociales» usualmente encaja en el de «persona miembro de una ONG». Un ejemplo de este tipo de respuesta sería el siguiente:

Óscar, indica que 2 es la más probable «No es que sea más probable, sino que es la más lógica porque si le interesan las cosas sociales, una ONG sería interesante para él. Depende también si le importan esas cuestiones lo suficiente como para luchar y defenderlas.»

Sin embargo, esto no significa que los estudiantes no conozcan la teoría, puesto que, cuando se les plantea la cuestión en un contexto académico (ítem 6), la gran mayoría de los estudiantes ( $85 \%$ ) contesta correctamente. Nótese que la situación planteada en el ítem 6 es similar a la del ítem 5: se trata de comparar las probabilidades de los sucesos $A$ y $A \cap B$. Si embargo, los contextos respectivos son bien distintos. Ahora debe razonarse de modo abstracto acerca de las probabilidades de $A$ y de
$A \cap B$. El objetivo de este ítem 6 es mostrar que el alumno puede ser capaz de razonar en términos formales cuando se elimina del enunciado el componente que sugiere emplear la experiencia personal acerca de los perfiles de individuos y poblaciones. Esto implicaría que la dificultad de aprendizaje no se sitúa en este caso en la parte del cálculo o en la aplicación mecánica de unas definiciones operativas sino en la interpretación de un enunciado desde el punto de vista probabilístico y en los ejercicios de abstracción y análisis.

En el ítem 7 se identifican los sucesos objeto de estudio y el estudiante debe indicar cuál de los dos sucesos es más probable, así como explicar su razonamiento. El primer objetivo de este ítem es detectar la creencia irreflexiva en la equiprobabilidad cuando se intenta aplicar el procedimiento normativo necesario para el cálculo de la probabilidad de un suceso. El segundo es detectar si el alumno concibe la probabilidad de un suceso según lo fácil que resulte generar ejemplos en los que tal suceso se cumple. Los resultados obtenidos aparecen en la tabla V.

Tabla V

Porcentajes de respuesta en el ítem 7.

\begin{tabular}{|l|c|}
\hline Categoría de respuesta & \% de respuestas $(\mathbf{N}=\mathbf{7 5})$ \\
\hline Respuesta correcta & 30,7 \\
\hline Heurístico de la accesibilidad & 28 \\
\hline Sesgo de la equiprobabilidad & 32 \\
\hline Respuesta incodificable & 5,3 \\
\hline En blanco & 4 \\
\hline
\end{tabular}

En la categoría «Respuesta correcta» se han agrupado aquellas respuestas que marcan $(d)$ y explican que no se conoce el número de palabras que existen de cada tipo. También se considerará correcta si indica que podría estimarse la probabilidad extrayendo aleatoriamente un gran número de palabras.

Sin embargo, casi un tercio de las respuestas (28\%) estima la probabilidad de un suceso según lo fácil que resulte generar o acceder a ejemplos en los que tal suceso se cumple o de recordar situaciones en las que se cumplió (heurístico de la accesibilidad). La forma de razonar que se encuentra en estas explicaciones está fuera del marco teórico y no se plantea el análisis formal del fenómeno aleatorio. Un ejemplo de este tipo de respuesta es la que marca Juan (respuesta $a$ ):

«Es más que nada pura lógica, puesto que es más probable que esté en la primera letra porque se puede coger en el diccionario las palabras que empiezan por $R$, que seguramente son muchas más que las que tienen $R$ en la tercera posición.»

Existe otro tercio de respuestas que trata de analizar el problema dentro del marco teórico explicado en clase, 
pero indican que, al ser dos las posibilidades que pueden ser obtenidas de forma aleatoria (la $R$ en primera o en tercera posición), ambas deben contar con la misma probabilidad de ocurrencia. En consecuencia, la respuesta elegida típicamente será $c$. Ejemplos de este tipo de respuesta:

María, marca la respuesta $c$. «Las variaciones que se pueden hacer en el resto de espacios libres es la misma.»

Jesús, marca la respuesta $c$. «Buscando una palabra en el diccionario creo que la probabilidad de que salga la $R$ en la primera o en la tercera es igual.»

Los resultados del ítem 8 son coherentes con los anteriores. En este caso no se trata de estimar una probabilidad sino de evaluar el número de posibilidades (512 para ambos esquemas) y en un contexto más próximo a un ejercicio académico. Probablemente por ello el número de respuestas correctas aumenta levemente hasta el $38 \%$. Sin embargo, nos encontramos con un $31 \%$ de respuestas que se encuentran en la categoría «Heurístico de la accesibilidad» porque estiman el número de trayectorias atendiendo al aspecto de ambas figuras, y otro $30 \%$ se reparte entre errores procedimentales cometidos al tratar de calcular el número de trayectorias y respuestas inclasificables.

\section{¿Cómo interpretan los estudiantes el concepto de probabilidad de un fenómeno aleatorio?}

Los ítems 9 y 10 tienen como objetivo estudiar qué significado atribuyen los alumnos a un valor de probabilidad dado para detectar el enfoque del resultado aislado. En el ítem 9 el estudiante debe razonar si es o no posible, con los datos disponibles, tomar una decisión acerca de cuál de las tres oficinas de inversión es más precisa en sus estimaciones de probabilidad. Los resultados obtenidos para el ítem 9 se indican en la tabla VI.

En la categoría «Respuesta correcta» se han agrupado aquellas respuestas que hacen referencia a lo insuficiente de la muestra y concluyen que no es posible tomar una decisión razonada probabilísticamente acerca de qué oficina de inversiones ofrece las estimaciones más precisas. La mayoría de las respuestas (casi el 60\%) se ha agrupado en la categoría «Enfoque del resultado aislado». En estas respuestas se interpreta el valor de la probabilidad como un pronóstico hacia el resultado de la siguiente ejecución del experimento aleatorio, siguiendo un razonamiento que está fuera de un análisis formal dentro del marco teórico explicado en clase. Así, en la situación planteada, el estudiante percibe una discrepancia entre la «predicción» de la oficina para cada empresa y el resultado real, y concluye que «la predicción fue errónea» o «la predicción fue acertada». En concreto, una explicación típica de esta categoría indica que las estimaciones de SANDAX se consideran poco precisas ( «no acertaron en ningún caso»); las de RICH INC, muy precisas («acertaron en los tres casos»); y las de MONEY SURE no se considerarán precisas ni imprecisas. Veamos un ejemplo de respuesta:
Tabla VI

Porcentajes de respuesta en el ítem 9.

\begin{tabular}{|l|c|}
\hline Categoría de respuesta & \% de respuestas $(\mathbf{N}=\mathbf{7 5})$ \\
\hline Respuesta correcta & 13 \\
\hline Enfoque del resultado aislado & 58,4 \\
\hline Sesgo determinista & 6 \\
\hline Respuesta incodificable & 4 \\
\hline En blanco & 18,6 \\
\hline
\end{tabular}

«Sí. Puede descartar a SANDAX, porque los resultados no han sido acertados. Se puede arriesgar con RICH INC y pensar que ha acertado o que se lo han dicho para que compre acciones. El más seguro es MONEY SURE, ya que es más prudente a la hora de hacer los cálculos.»

Existe una pequeña minoría de respuestas $(6 \%)$ que indica que no es posible obtener ninguna conclusión dada la complejidad de la situación planteada, en este caso de la bolsa, mostrando una concepción calificada como causal de los fenómenos aleatorios que ya hemos comentado en los ítems 1 y 2 y en la primera situación para entrevista.

Cuando a los estudiantes se les plantea la misma cuestión de interpretar el valor de la probabilidad de un suceso en un contexto más cotidiano y menos académico (ítem 10), el número de respuestas de la categoría «Enfoque del resultado aislado» aumenta hasta comprender casi las tres cuartas partes $(74,3 \%)$. Estas explicaciones analizan la cuestión fuera del marco teórico e interpretan incorrectamente el valor de la probabilidad como un pronóstico acerca del resultado de la siguiente ejecución del experimento aleatorio en vez de interpretarlo como una característica aproximada de la población, que es solamente válida para amplias muestras tomadas de forma aleatoria. En este sentido y coherentemente con explicaciones al margen del cuerpo teórico, no se tiene en cuenta el tamaño de la muestra de que se dispone.

Estas explicaciones alternativas de la probabilidad como pronóstico acerca de lo que ocurrirá la próxima vez que se ejecute el experimento aleatorio se muestran claramente también a través de entrevistas personales a los alumnos. El siguiente fragmento corresponde a una entrevista de la situación problemática 3 en la que Jaione debía interpretar el valor de $p=0,7$ de probabilidad de lluvia que el meteorólogo había estimado para mañana.

- Fragmento de entrevista correspondiente a la situación problemática 3

Jaione: «Que es posible que mañana llueva.»

Entrevistador: «Pero ese valor, el 0,7, ¿cómo lo interpretas?» $J .:$ «Que es más probable que llueva a que no.»

$E .:$ «Imagina que tienes que tomar alguna clase de decisión para mañana. Por ejemplo, te dedicas a pintar fachadas y no 
puedes trabajar si llueve. ¿Crees que ese valor de 0,7 puede servirte para algo?»

$J .:$ «Yo pensaría que lo más seguro es que llueva, pero tampoco es seguro.»

E.: «Y para que pienses que existe cierta seguridad, ¿qué valor de probabilidad tendrían que darte?»

$J .:$ «Como un $90-95 \%$.»

Obsérvese que esta alumna percibe claramente la situación de incertidumbre, pero interpreta el valor $p=0,7$ como una expectativa acerca de si mañana lloverá, sin la menor referencia a un significado frecuencial.

Como se sabe, según esta interpretación de la probabilidad como pronóstico, si una probabilidad se acerca a los extremos 0 o 1 , el suceso se considera imposible o seguro, respectivamente; y sólo si se acerca al 0,5, en el que se considerará verdaderamente aleatorio. Nosotros hemos intentado, a través de estas entrevistas personales, precisar en mayor medida cuál es el subintervalo donde el alumno detecta la mayor incertidumbre. El siguiente fragmento, que corresponde a la misma situación problemática del fragmento anterior, Javier muestra también una interpretación de la probabilidad como pronóstico y además es capaz de especificar dicho intervalo:

- Fragmento de entrevista correspondiente a la situación problemática 3

Javier: «Con esa probabilidad de que llueva, lo más seguro es que llueva.»

Entrevistador: «¿Y si la probabilidad de lluvia fuera de un 30\%?»

$J .:$ «Entonces casi con seguridad iba a hacer buen tiempo.»

$E .:$ «Intenta indicar un intervalo dentro de 0,1 en el que ves una gran incertidumbre acerca de lo que va a ocurrir mañana.»

$J$. : «A partir del mismo 0,7, empezaría a preocuparme, con 0,8 casi seguro que llueve y de ahí en adelante...»

$E .:$ «YY hacia la izquierda?»

$J .:$ «Del 0 al 0,3 prácticamente la probabilidad de que llueva es nula.»

Los resultados obtenidos coinciden en parte con los indicados en la bibliografía sobre esta concepción (Konold, 1991; Serrano et al., 1998). En ellos se indica que esta concepción incorrecta da lugar a un pronóstico exacto acerca del resultado de la próxima ejecución, de tal modo que en la situación del ítem 10 la probabilidad de 0,7 se interpretaría como «el paciente sobrevivirá». Sin embargo, nosotros hemos detectado en estudiantes universitarios que a partir del dato ofrecido de probabilidad se valoran las expectativas. Así, en el ítem 10 la probabilidad de 0,7 no se interpreta de forma tan explícita sino que, a partir del dato ofrecido de probabilidad, se valoran las expectativas de supervivencia para ese paciente específico.

En los ítems 11 y 12 se presentan situaciones en las que los estudiantes tienen que analizar si es posible estimar de forma precisa el número de ocurrencias de un suceso cuya probabilidad es conocida a partir de la muestra disponible. El objetivo de ambos ítems es detectar si el alumno cree que es posible estimar de forma precisa el número de ocurrencias de un suceso cuya probabilidad es conocida independientemente del tamaño de la muestra (insensibilidad al tamaño de la muestra).

En el ítem 11 se presenta una situación cotidiana algo alejada del contexto académico. El estudiante debe razonar si es posible emitir algún juicio acerca de cómo de precisas son cada una de las estimaciones de la probabilidad de un suceso. Los resultados obtenidos se muestran en la tabla VII.

Tabla VII

Porcentajes de respuesta en el ítem 11.

\begin{tabular}{|l|c|}
\hline Categoría de respuesta & \% de respuestas $(\mathbf{N}=\mathbf{3 5})$ \\
\hline Respuesta correcta & 8,6 \\
\hline $\begin{array}{l}\text { Insensibilidad al tamaño } \\
\text { de la muestra }\end{array}$ & 85,6 \\
\hline Respuesta incodificable & 2,9 \\
\hline En blanco & 2,9 \\
\hline
\end{tabular}

Una pequeña cantidad de estudiantes $(8,6 \%)$ analiza correctamente la situación. Explican que, de acuerdo con el marco teórico, el análisis lleva a la misma respuesta para los tres hospitales: la probabilidad de supervivencia ofrece una estimación del número de pacientes que sobrevivirán de entre una muestra amplia y, por tanto, ninguna conclusión se obtiene acerca de cómo de precisas son las estimaciones de los hospitales contando con una muestra de cinco datos.

Sin embargo, la gran mayoría de las respuestas $(85,6 \%)$ se agrupa en la categoría «Insensibilidad al tamaño de la muestra». En estas explicaciones, los estudiantes no presentan una perspectiva frecuencial de la probabilidad. En los razonamientos de esta categoría se muestra un juicio acerca de cómo de precisas son las estimaciones calculando explícita o implícitamente los «valores esperados» del número de pacientes de la muestra de cinco, que «deberían recuperarse» en caso de ser precisas las estimaciones de los hospitales. Ejemplos de respuesta de esta categoría son:

«Las estimaciones son bastante precisas. Si calculamos el $20 \%$, el $50 \%$ y el $70 \%$ de los cinco, obtenemos, más o menos haciendo ajuste, los enfermos recuperados, aunque en Cruces la estimación no es muy buena.»

«Las estimaciones de probabilidades de curación del hospital de Txagorritxu son perfectas; las de Aranzazu, buenas, se curaron más de los que se estimaban; y las estimaciones del hospital de Cruces fueron malas, pues se recuperaron menos de los que se estimaba.»

También se han recogido en esta categoría razonamientos del tipo «el pasado condiciona el futuro» (Muñoz, 1998), como puede ser el siguiente: 
«En observaciones anteriores se debió dar una mayor supervivencia, que ahora se compensa con una supervivencia menor de lo esperado».

Aquí también se da una insensibilidad al tamaño de la muestra, puesto que, para que cinco nuevas observaciones «compensen» las anteriores, la muestra total debe ser de muy pequeño tamaño y además debe pensarse que la relación entre los pacientes de la muestra que se recuperaron y el tamaño de la muestra permanece casi constante, independientemente de la muestra.

Esta concepción alternativa de la probabilidad que no toma en consideración el tamaño muestral también se constata a través de entrevistas personales a los alumnos. El siguiente fragmento corresponde a una entrevista en la que a Amaia se le planteó la situación problemática 4. Nótese que la alumna encuentra en la pequeña muestra disponible (6 elementos) razones suficientes para dudar de la estimación ofrecida del $50 \%$.

\section{- Fragmento de entrevista correspondiente a la situa- ción problemática 4}

Amaia: «En realidad no es cierto el dato del 50\%.»

Entrevistador: «Bien, pero ahora imagina que es absolutamente cierto el dato, comprobado ante notario, que realmente se empaqueta el 50\% de los packs con premio. ¿Qué pensarías?»

$A:$ Q Que tengo mala suerte. Se supone que tienen que salir tres premios... Se habrán quedado en otros seis packs...»

$E:$ « Y si quieres otros dos peluches?»

A: «Compraría otros dos packs.»

$E .:$ «Y qué esperarías?»

A.: «Un premio en cada pack».

E.: «Pero lo que hay en esos dos packs nuevos que vas a comprar no resulta afectado por lo que ya has comprado. ¿Cómo te cuadra eso?»

\section{A.: [No contesta, no sabe dar una explicación coherente.]}

Cuando a los estudiantes se les asegura que la estimación del $50 \%$ es cierta, como es el caso de la entrevista, siguen pensando en términos alternativos al concepto frecuencial formal de probabilidad y explican la discrepancia entre el «valor esperado». (tres packs premiados) y el valor real (sólo uno premiado) mediante la idea de que «el pasado condiciona el futuro».

«Se supone que tienen que salir tres premios... Se habrán quedado en otros seis packs... Comprando otros dos packs, espero premio en los dos...»

Los resultados del ítem 12 son convergentes con el ítem 11 y las entrevistas. El ítem 12 es similar al ítem 11, pero cambiando el contexto hospitalario por uno industrial y, por tanto, más familiar al contexto académico. Esto quizá explique que el número de respuestas correctas aumente algo $(28 \%)$, pero siguen siendo mayoría (65\%) las respuestas que se sitúan en la categoría «Insensibilidad al tamaño de la muestra».

\section{CONCLUSIONES}

Se observa una clara convergencia entre los resultados de este trabajo con estudiantes universitarios y otras investigaciones del área en enseñanza secundaria. Sin embargo, también existen algunas diferencias que ya hemos comentado en el apartado anterior.

De los resultados obtenidos podemos deducir que, de acuerdo con la hipótesis emitida, la mayoría de los estudiantes presenta, tras su formación, ideas alternativas a las formales sobre los fenómenos aleatorios, el modo de estimar la probabilidad y la forma de interpretar su valor. Es de resaltar que una gran parte de los alumnos contesta correctamente cuando se trata de aplicar un procedimiento memorístico a una cuestión similar a las realizadas en clase. Éste sería en caso del ítem 6 y, en menor medida, el del ítem 8, pero también reflejaría esta idea el alto índice de aprobados que se contabiliza en los exámenes oficiales de $2^{\circ}$ curso de Ingeniería Técnica, en que los estudiantes deben resolver cuestiones de corte también similar. Sin embargo, el objetivo de nuestros diseños ha sido analizar las respuestas de los alumnos cuando les son planteadas situaciones de alta demanda cognitiva, y en este sentido se produce un alto fracaso que indica un aprendizaje no comprensivo de los conceptos. Para finalizar, intentaremos sintetizar los resultados obtenidos con relación a los sesgos y heurísticos planteados.

\section{1) Sesgo determinista}

El sesgo determinista ha sido investigado en este trabajo mediante tres vías: $a$ ) discusión de una propuesta de solución determinista (ítem 2); b) buscando respuestas del tipo «el fenómeno no puede estudiarse porque se trata de azar» (ítem 1); y c) detectando confusión entre condicionamiento y causación (primera situación problemática para entrevista). Una parte significativa de estudiantes, más de un tercio $(38,8 \%$ en el ítem 2$)$, no percibe el carácter aleatorio de las situaciones presentadas y tiende a proponer soluciones deterministas. La prevalencia abusiva del pensamiento causal sobre el probabilístico también se manifiesta de forma significativa en el ítem 1 (20\%). Estos resultados se confirman en las entrevistas, en las que todos los estudiantes entrevistados presentaron confusión entre la independencia causal y la estadística y no relacionaron la situación planteada con el concepto de probabilidad condicionada, ampliamente trabajado en clase.

\section{2) Heurístico de representatividad}

En cuanto a las ideas de los alumnos acerca de cómo se configuran las secuencias aleatorias, una gran mayoría de ellos (más del $80 \%$ de las respuestas de los ítems 3 y 4) piensa que una secuencia aleatoria que muestre algún tipo de simetría, patrón o regularidad de cualquier tipo es más improbable que cualquier otra de idéntico tamaño pero que no presente estructura reconocible alguna. Esta gran mayoría de los alumnos no toma en consideración un factor fundamental en la concepción frecuencial de la probabilidad, el tamaño muestral, y piensa que el valor 
de la probabilidad permite estimar de forma muy precisa el número de ocurrencias del suceso, aun en muestras pequeñas (más del $85 \%$ en el ítem 11).

Respecto a las explicaciones acerca de los procedimientos para estimar la probabilidad, cuando se trata de un contexto cotidiano no académico, más de los dos tercios (77\% de las respuestas del ítem 5) acude a una concepción subjetiva de lo que es representativo de una población, sin ningún intento de análisis formal. Sin embargo, algo muy diferente ocurre cuando es un contexto idéntico al trabajado en clase. Entonces la mayoría de los estudiantes es capaz de aplicar un análisis formal dentro del marco teórico de la teoría de la probabilidad (85\% en el ítem 6). Notamos de nuevo la importancia de la interacción sujeto-tarea y la dificultad de los estudiantes para realizar un ejercicio de abstracción.

\section{3) Heurístico de accesibilidad}

Se ha detectado el uso de este heurístico en porcentajes similares en los ítems 7 y $8(28 \%$ y $31 \%$ respectivamente). Es significativo el porcentaje de alumnos que reconoce una situación familiar en la del ítem 8 y trata de aplicar un procedimiento de cálculo combinatorio, pero comete algún error (14\%).

\section{4) Sesgo de equiprobabilidad}

Se han utilizado los ítems 1 y 7 para detectar la creencia en la equiprobabilidad de todos los resultados del experimento (elementos del espacio muestral). Se observa en porcentajes muy diferentes en los ítems empleados (52\% en el ítem 1 y $32 \%$ en el ítem 7). Un porcentaje significativo de alumnos que mostró el sesgo de equiprobabilidad en el ítem 1 opta, en cambio, por otra estrategia diferente en el ítem 7 (ya sea una estrategia normativa o bien sugerida por el heurístico de accesibilidad) a pesar de que podrían aplicar la misma. Así pues, el uso de cierto sesgo por parte de un individuo puede o no verse desplazado en favor de algún otro sesgo o de una estrategia normativa cuando se plantean diferentes situaciones

\section{5) Enfoque del resultado aislado}

En el ítem 9 se ha detectado, en una mayoría de las respuestas (casi en un 60\%), una interpretación de la probabilidad como pronóstico hacia la siguiente ejecución del experimento. Pero cuando los estudiantes deben

\section{REFERENCIAS BIBLIOGRÁFICAS}

AHLGREN, A. y GARFIELD, J. (1991). Analysis of the Probability Curriculum, en Kapadia, R. y Borovenik, M. (eds.), Chance encounters: probability in education, pp. 107-134. Netherlands: Kluwer Academic Publishers. interpretar el valor de la probabilidad de un suceso en un contex to cotidiano poco académico (ítem 10), el número de respuestas propias de este enfoque aumenta hasta comprender casi las tres cuartas partes $(74,3 \%)$. Además, la interpretación de los alumnos no es tan explícita como puede ser «el paciente sobrevivirá», sino que se valoran las expectativas para ese paciente específico. En las entrevistas personales con la tercera situación problemática, los alumnos ofrecen más detalles de esta valoración y establecen intervalos que, a su juicio corresponden a una mayor o menor incertidumbre.

Se ha encontrado que sólo una minoría de estudiantes universitarios (alrededor del 10\%) analiza los fenómenos aleatorios propuestos desde un punto de vista formal de la teoría de la probabilidad y utiliza correctamente los procedimientos necesarios para el cálculo de la probabilidad de un suceso. Se supone que en este grupo tendría que encontrarse la mayoría de los estudiantes universitarios después de seguir varios cursos de estadística en secundaria y el curso de introducción a la teoría de la probabilidad en la universidad; sin embargo, los resultados obtenidos muestran otra realidad bien diferente. Parecen existir serias dificultades de aprendizaje tanto en el campo de las concepciones sobre los fenómenos aleatorios y la probabilidad (dificultades ontológicas, conceptuales y metodológicas) como en el campo de los procedimientos normativos necesarios para calcular la probabilidad de un suceso (dificultades procedimentales). Si a estas dificultades añadimos los efectos de una enseñanza basada en la exposición aproblemática del cuerpo teórico actual sin tener en cuenta los saltos cualitativos que se dieron en la teoría, y que presenta los conceptos únicamente en su forma operativa final, se pueden entender las dificultades que tienen los estudiantes en esta teoría.

Los resultados obtenidos nos llevan a afirmar que los fenómenos aleatorios no presentan explicaciones «naturales» ni sencillas de dar y que no constituyen fenómenos elementales que se pueden impartir de forma rápida sin detenerse a analizarlos de acuerdo con una teoría que ocupó a los matemáticos durante tres siglos. Así pues, el conocimiento de las categorías explicativas nos pueden ayudar a diseñar materiales y estrategias de enseñanza que puedan facilitar un cambio conceptual, ontológico y metodológico. Éste será el objetivo de nuestros próximos trabajos.
BATANERO, C., NAVARRO-PELAYO, V. y GODINO, J. (1997). Effect of the implicit combinatorial model on combinatorial reasoning in Secondary School pupils. Educational Studies in Mathematics, 32(2), pp. 181-199. 
BATANERO, C. (2000). ¿Hacia dónde va la educación estadística? Blaix, 15, pp. 2-13.

BOROVCNIK, M., BENTZ, H. J. y KAPADIA, R. (1991). A Probabilistic Perspective, en Kapadia, R. y Borovenik, M., (eds). Chance encounters: probability in education, pp. 27-70. Netherlands: Kluwer Academic Publishers.

BOROVCNIK, M. y BENTZ, H. J. (1991). Empirical Research in Understanding Probability, en Kapadia, R. y Borovenik, M. (eds.). Chance encounters: probability in education. Netherlands: Kluwer Academic Publishers, pp. 73-105.

BOROVCNIK, M. y PEARD, R. (1996). Probability, en Bishop, A.J., Clements, K., Keitel, C., Kilpatrick, J. y Laborde, C. (eds). International Handbook of Mathematics Education, pp. 239-287. Netherlands: Kluwer Academic Publishers.

CANAVOS, G. C. (1992). Probabilidad y estadística, p. 29 Madrid: McGraw-Hill.

DÍAZ, J., BATANERO, C. y CAÑIZARES, M.J. (1996). Azar y probabilidad. Madrid: Síntesis

FALK, R. (1983). Experimental Models for Resolving Probabilistic Ambiguities. Proc. Seventh Int. Conf. Psychology of Mathematics Education. Rehovot.

GNEDENKO, B. (1996). Teoría de las probabilidades, p. 27. Madrid: Rubiños.

GÓMEZ, P. y PERRY, P.I. (eds). (1996). La problemática de las matemáticas escolares. Mexico: Grupo Editorial Iberoamericana.

GRUPO AZARQUIEL (1996). Proyecto Azarquiel Matemáticas. Madrid: Ediciones de la Torre.

GUISASOLA, J. y BARRAGUÉS, J.I. (1999). Dificultades de aprendizaje de conceptos relativos al azar y la probabilidad en estudiantes de Ingeniería Técnica Industrial. Seminario Interfacultades sobre Enseñanza-Aprendizaje de la Estadística. San Sebastián: Servicio de Publicaciones de la EUITI.

HIRSCH,L. S. y O'DONNELL, A. M. (2001). Representativeness in Statistical Reasoning: Identifying and Assessing Misconceptions. Journal of Statistics Education, 9(2).

KAHNEMAN, D. y TVERSKY, A. (1972). Subjetive Probability: a judgment of representativeness. Cognitive Psychology, 5 , pp. $430-454$

KAHNEMAN, D., SLOVIC, P. y TVERSKY, A. (1982). Judgment under uncertainty: heuristics and biases. Nueva York: Cambridge University Press.

KAPADIA, R. (1984). Children's Intuitions and Conceptions of Probability. Londres: Polytechnic of the South Bank.

KONOLD, C. (1991). Understanding student's beliefs about probability, en Glasersfeld, E. V. (ed.). Radical Constructivism in Mathematics Education, pp. 139-156. Dordrecht: Kluwer.

LECOUTRE, M.P. (1985). Effect d'informations de nature combinatoire et de nature frécuentielle sur les jugements probabilistes. Recherches en Didactique des Mathématiques, 6, pp. 193-213.

LECOUTRE, M.P. (1992). Cognitive models and problem spaces in «purely random» situations. Educational Studies in Mathematics, 23, pp. 557-568.

LECOUTRE, M.P. y DURAND, J.L. (1988). Jugements probabilistes et modèles cognitifs: étude d'une situation aléatoire. Educational Studies in Mathematics, 19, pp. 357-368.

LECOUTRE, M.P. y CORDIER, J. (1990). Effect du mode de présentation d'un problème aléatoire sur les modèles développés par les élèves. Bulletin de l'APMEP, 372, pp. 9-22.

MUÑOZ, A. (1998). Algunas ideas preconcebidas sobre probabilidad. Suma, 29, pp. 29-34.

PAULOS, J.A. (1995). Bosnia: ¿Vietnam o la segunda guerra mundial? Disponibilidad psicológica y efectos ancla. Un matemático lee el periódico, pp. 31-36. Barcelona: Tusquets Editores.

POZO, J.I., (1999). Más allá del cambio conceptual: el aprendizaje de la ciencia como cambio representacional. Enseñanza de las Ciencias, 17(3), pp. 513-520.

SÁENZ, C. (1998). Teaching Probability for Conceptual Change. Educational Studies in Mathematics, 35(3), pp. 233-254.

SCHOLZ, R.W. (1991). Psychological Research in Probabilistic Understanding, en Kapadia, R. y Borovcnik, M. (eds.), Chance encounters: probability in education, pp. 213-253. Netherlands: Kluwer Academic Publishers.

SERRANO, L., BATANERO, C. y ORTÍZ, J.J. (1996). Interpretación de enunciados de probabilidad en términos frecuenciales por alumnos de bachillerato. Suma, 22, pp. 43-49.

SERRANO, L., BATANERO, C., ORTÍZ, J. J., y CAÑIZARES, M.J. (1998). Heurísticas y sesgos en el razonamiento probabilístico de los estudiantes de secundaria. Educación Matemática, 22, pp. 7-25

SERRANO, L., BATANERO, C. y CAÑIZARES, M. J. (1999). Concepciones sobre distribuciones aleatorias planas en alumnos de secundaria. Epsilon, 43-44, pp. 149-162.

SERRANO, L., BATANERO, C., ORTÍZ, J. J., y CAÑIZARES, M.J. (2001). Concepciones de los alumnos de secundaria sobre modelos probabilísticos en las secuencias de resultados aleatorios. Suma, 36, pp. 23-32.

SOLSONA, N., IZQUIERDO, M. y GUTIÉRREZ, R. (2000). El uso de razonamientos causales en relación con la significación de los modelos teóricos. Enseñanza de las Ciencias, 18(1), pp. 15-23.

STEWART, I. (1998). Una ramera llamada fortuna. De aquí al infinito, pp. 181-195. Barcelona: Drakontos.

WHITE, R. y GUNSTONE, R. (1992). Probing Understanding. Londres: The Palmer Press. 


\section{ANEXO}

\section{CUESTIONARIO}

- Ítem 1. Un agente de bolsa ha comprado acciones de tres empresas. ¿Cuál es la probabilidad de que en el transcurso de un mes suban las acciones de al menos dos de las tres empresas?

a) La probabilidad es $1 / 2$.

b) La probabilidad es $3 / 8$.

c) La probabilidad es

d) No puedo calcular la probabilidad.

Por favor, razona tu respuesta.

- Ítem 2. Tu amigo Borja es muy aficionado a los experimentos físicos. Te ha llamado para hablarte del último que tiene entre manos. Sobre un panel vertical ha clavado algunos clavos, como aparece en la siguiente figura:

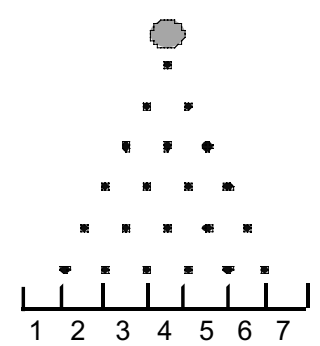

Cuando se deja caer la bola desde la parte superior, rebota por los clavos y termina en alguno de los agujeros de la parte inferior. Lo que intenta Borja es predecir cuál será el agujero en el que entrará la bola. Ha medido con mucho cuidado la posición de los clavos, el peso y el diámetro de la bola y las características del material con que está fabricada. Su idea es emplear todos estos datos y los principios y las leyes de física sobre choques y movimiento de objetos para calcular la trayectoria que seguirá la bola en su caída, y así poder predecir en qué agujero entrará.

Sin embargo, Borja no ve claro cómo ponerse en marcha, qué leyes físicas emplear y cómo hacerlo. Por eso te ha pedido ayuda. Se trata de que le aconsejes de la siguiente forma:

a) Te ha explicado su modo de enfocar el problema. Explícale qué te parece ese enfoque.

b) Explícale los pasos más importantes que piensas que habría que dar para resolver el problema.

- Ítem 3. Ha llegado a oídos de la policía el «soplo» de que las ruletas del casino CANTEEN CLuB están amañadas. Para investigar el caso, un agente de incógnito ha jugado ocho veces en cada una de las cinco ruletas del casino y ha anotado si salió rojo $(R)$ o negro $(N)$. He aquí los datos que trajo el agente:

\begin{tabular}{|c|c|}
\hline Número de ruleta & Secuencia de ocho jugadas de esa ruleta \\
\hline 1 & RRRRNNNN \\
\hline 2 & RRNNNRNR \\
\hline 3 & RNRNRNRN \\
\hline 4 & NNRNRRNN \\
\hline 5 & NNNNNNNN \\
\hline
\end{tabular}

Antes de abrir una investigación, la policía necesita saber si existen indicios de que, efectivamente, la ruleta está amañada y que el color ganador no es aleatorio sino que en realidad es elegido de antemano por el crupier.

Por favor, indica con un número del 0 al 4 tu grado de confianza en que la secuencia de colores de cada ruleta salió al azar. Así:

Confianza 0: Creo que la secuencia de esa ruleta NO fue obtenida de forma aleatoria

Confianza 1: Creo que existen indicios de que la secuencia de esa ruleta NO fue obtenida de forma aleatoria

Confianza 2: Indecisión. No puedo inclinarme hacia ninguna de las posibilidades.

Confianza 3: Creo que existen indicios de que la secuencia de esa ruleta Sí fue obtenida de forma aleatoria

Confianza 4: Creo que la secuencia de esa ruleta Sí fue obtenida de forma aleatoria 
A la ruleta 1 le doy una confianza de por la siguiente razón: A la ruleta 2 le doy una confianza de por la siguiente razón:

A la ruleta 3 le doy una confianza de por la siguiente razón: A la ruleta 4 le doy una confianza de por la siguiente razón: A la ruleta 5 le doy una confianza de por la siguiente razón:

- Ítem 4. En una sala de juegos de azar se han instalado tres máquinas del modelo MAGIC PUSH. Esta máquina muestra un panel de cinco filas de cinco casillas cada una, como aparece en la figura:

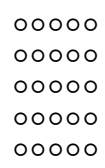

El jugador debe elegir una casilla de entre las veinticinco. Entonces, la máquina seleccionará al azar cinco casillas de entre las veinticinco y, si entre esas cinco está la que eligió el jugador, éste gana. El gerente de la sala, para probar las máquinas, ha jugado tres veces en cada una de ellas. La siguiente figura muestra qué cinco casillas fueron elegidas por cada máquina en cada una de las tres jugadas:

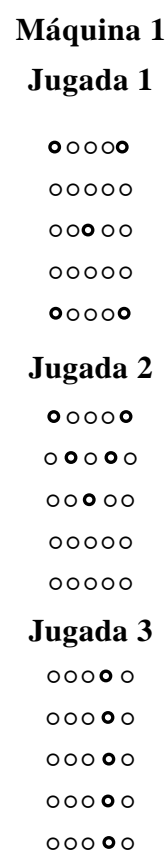

Máquina 2
Jugada 1
○००००
०००००
०००००
०००००
०००००

Jugada 2

00000

00000

00000

00000

00000

Jugada 3

00000

00000

00000

00000

00000

\section{Máquina 3 \\ Jugada 1 \\ 00000 \\ 00000 \\ 00000 \\ 00000 \\ 00000}

Jugada 2

०००००

00000

00000

00000

00000

Jugada 3

00000

00000

00000

00000

०००००

Al ver los resultados, el gerente duda de que las máquinas elijan las casillas de forma aleatoria. ¿Qué crees tú? Para cada una de las máquinas debes indicar con qué confianza crees que las casillas sí han sido elegidas al azar por la máquina. Así:

Confianza 0: Creo que esa máquina NO elige de forma aleatoria las casillas.

Confianza 1: Creo que existen indicios de que esa máquina NO elige de forma aleatoria las casillas.

Confianza 2: Indecisión. No puedo inclinarme hacia ninguna de las posibilidades.

Confianza 3: Creo que existen indicios de que esa máquina Sí elige las casillas de forma aleatoria.

Confianza 4: Creo que esa máquina SÍ elige de forma aleatoria las casillas.

A la máquina 1 le doy una confianza de A la máquina 2 le doy una confianza de A la máquina 3 le doy una confianza de por la siguiente razón: por la siguiente razón: por la siguiente razón: 
- Ítem 5. R.M. es una persona joven, soltera, abierta, brillante, universitaria y muy interesada en las cuestiones sociales. ¿Cuál de las situaciones ( 1 o 2 ) te parece más probable?

1) R. M. trabaja en un banco.

2) R. M. trabaja en un banco y es miembro de una ONG.

a) La situación 1 es la más probable.

b) La situación 2 es la más probable.

c) Ambas situaciones tienen la misma probabilidad.

d) Otra respuesta.

Por favor, razona tu respuesta (Tomado de bibliografía)

- Ítem 6. Sean A y B dos sucesos. Razonar si la siguiente relación es cierta: $\mathrm{p}(\mathrm{A} \cap \mathrm{B}) \leq \mathrm{p}(\mathrm{A})$

- Ítem 7. Tomemos aleatoriamente del diccionario una palabra que contenga una vez la letra $R$, ya sea en su primera o en su tercera posición. Elige una respuesta:

a) Es más probable que la $R$ se encuentre en la primera posición.

b) Es más probable que la $R$ se encuentre en la tercera posición.

c) Es tan probable que la $R$ se encuentre en primera posición como que se encuentre en la tercera.

d) No puedo determinar cuál de las posiciones de la $R$, primera o tercera, es más probable.

Por favor, razona tu respuesta (Tomado de Bibliografía)

- Ítem 8. Observa los esquemas 1 y 2, formados por varias filas de círculos. En cada esquema, se trata de pasar de un círculo cualquiera de la primera fila a un círculo cualquiera de la última fila, de tal modo que, desde un círculo, sólo puede saltarse a otro cualquiera pero que esté en la fila inmediatamente inferior.

$\begin{array}{ll}\text { Esquema 1 } & \text { Esquema } 2 \\ 00000000 & 00 \\ 00000000 & 00 \\ 00000000 & 00 \\ & 00 \\ & 00 \\ & 00 \\ & 00 \\ & 00 \\ & 00\end{array}$

La cuestión es: ¿En cuál de los dos esquemas ( 1 o 2 2) hay más formas posibles de efectuar el trayecto?

a) En el esquema 1 .

b) En el esquema 2.

c) Existe igual número de formas en ambos esquemas.

Por favor, razona tu respuesta (Tomado de bibliografía)

- Ítem 9. Un inversor debe decidir con qué oficina de inversiones trabajar, de entre tres que se le han ofrecido: SANDAX, MONEY SURE y RICH INC. Para ello, la semana pasada pidió a cada oficina una estimación de la probabilidad de que esta semana subieran las acciones de las empresas ACERINOX, TELEMATIC y RAUDi. Cada una de las tres oficinas dio al inversor su estimación de probabilidad de subida para cada una de las tres empresas, según aparece en la siguiente tabla.

\begin{tabular}{|c|c|c|c|}
\hline & $\begin{array}{l}\text { Probabilidad de que suban } \\
\text { las acciones de ACERINOX }\end{array}$ & $\begin{array}{l}\text { Probabilidad de que suban } \\
\text { las acciones de TELEMATIC }\end{array}$ & $\begin{array}{l}\text { Probabilidad de que suban } \\
\text { las acciones de RAUDI }\end{array}$ \\
\hline Según SANDAX & 0,1 & 0,2 & 0,25 \\
\hline Según M. SURE & 0,5 & 0,54 & 0,48 \\
\hline Según R. INC & 0,8 & 0,7 & 0,9 \\
\hline
\end{tabular}

Pues bien, resulta que esta semana las acciones de las tres empresas han subido. ¿Crees que el inversor tiene suficientes elementos de juicio para decidir con qué oficina de inversiones trabajar? ¿Por qué?

- Ítem 10. Un enfermo aquejado de aneurisma de aorta debe ser operado de inmediato en el hospital de Cruces. El jefe de la unidad coronaria explica a los familiares que los pacientes en estas condiciones cuentan con una probabilidad de 0,7 de sobrevivir a la operación. 
¿Cómo crees tú que deben interpretar los familiares ese dato que les ofrece el doctor?

- Ítem 11. Se han tomado al azar los historiales médicos de cinco enfermos de cada uno de los hospitales de Txagorritxu, Nuestra Señora de Aranzazu y Cruces, todos ellos con la misma dolencia pulmonar. En los historiales de los cinco enfermos de Txagorritxu consta que se estimó en 0,2 la probabilidad de curación. Para los cinco enfermos de Nuestra Señora de Aranzazu, la probabilidad fue de 0,5 , mientras que, para los cinco enfermos de Cruces, la probabilidad de curación fue 0,7.

Pues bien, de los cinco enfermos tratados en Txagorritxu, se recuperó sólo uno de ellos; de los cinco pacientes de Nuestra Señora de Aranzazu se recuperaron tres; y de los cinco pacientes de Cruces, se recuperaron dos. ¿Te es posible obtener alguna conclusión acerca de cómo de acertadas son las estimaciones de la probabilidad de supervivencia que se dan en los diferentes hospitales?

- Ítem 12. Según el jefe de mantenimiento de DAEwoo, el $70 \%$ de los motores eléctricos sufre alguna avería antes de las dos mil horas de funcionamiento. Tomamos cinco motores nuevos, los instalamos, y resulta que los cinco superan las dos mil horas de funcionamiento sin fallo. ¿Crees que se equivoca el jefe de mantenimiento? ¿Por qué?

\section{ANEXO \\ SITUACIONES PROBLEMÁTICAS PARA ENTREVISTAS}

Situación 1. Sobre la mesa tenemos cuatro cartas: dos ases y dos reyes. Las colocamos boca abajo y las revolvemos. Naturalmente, si ahora sacamos una carta al azar, la probabilidad de que salga $A S$ es idéntica a la probabilidad de que salga rey (esto es, 0,5$)$. Pues bien, lo que hacemos es extraer una carta al azar pero la apartamos sin mirar qué carta es. Luego, de entre las tres cartas restantes sacamos otra, y esa sí la descubrimos y resulta que es un as. Según este segundo resultado, ¿la probabilidad de que la primera carta haya sido un $A S$ es ahora mayor, igual o menor que 0,5 ?

Situación 2. Supongamos que tenemos un bombo con diez bolas numeradas, del 0 al 9. Agitamos el bombo, sacamos una bola, anotamos su número y luego la devolvemos al bombo. Hacemos la misma operación cinco veces. Piensa en estos tres resultados posibles: 22211, 12345 y 83056 . ¿Cuál de ellos te parece más probable? ¿Cuál el menos probable?

Situación 3. Supongamos que el meteorólogo de la ciudad asegura que existe una probabilidad de lluvia para mañana del $70 \%$. ¿Cómo interpretas esa afirmación?

Situación 4. Imagina que durante una promoción de Coca-Cola, la organización asegura que la mitad de los packs de doce botellas trae premio (peluches parlantes de la familia Simpson). Con la esperanza de colocar a Bart, Homer y Lisa sobre tu mesilla, has comprado seis packs de Coca-Cola. Y resulta que sólo uno de ellos ha traído premio, mientras que en los cinco restantes sólo has encontrado una tarjeta con el mensaje «Gracias por su compra. Siga buscando porque uno de cada dos packs trae premio». ¿Crees que hay en realidad menos del 50\% de packs con premio? 